\title{
Formulación de alternativas para mitigar los impactos ambientales causados por la refinería ilegal en Tibú
}

\section{Formulating Alternatives to Mitigate the Environmental Impacts of Illegal Refineries in Tibú}

Mayra A. Canesto A. ', Juan G. Téllez C. ${ }^{1}$

\section{Resumen}

Este documento tiene por objeto evidenciar una problemática relacionada con el hurto del crudo en Tibú, Norte de Santander, donde los Grupos Armados Organizados, por medio de válvulas instaladas ilegalmente a lo largo de los oleoductos de la región, provocan impactos ambientales que afectan la economía de las regiones y vulneran los derechos fundamentales de la población. Es por ello por lo que cabe preguntar: ¿cuáles alternativas serían las más viables de implementar para mitigar este estado de cosas? El abordaje teórico de algunas fuentes que se han ocupado de esta problemática, permitió dar respuesta a la anterior pregunta; es una investigación de tipo descriptivo comparativo, con enfoque inductivo, puesto que va de lo general a lo particular. Como alternativas para mitigar los impactos ambientales causados por la refinería ilegal, se plantearon diversas alternativas sociales, ambientales y de seguridad, según la conclusión a la que se llegó.

Palabras clave: impacto ambiental, petróleo, refinería ilegal.

\section{Abstract}

This paper seeks to highlight a problem related to the theft of crude oil in Tibú, Norte de Santander, where Organized Armed Groups (OAGs), through valves illegally

\footnotetext{
1 Especialización Gestión Ambiental y Desarrollo Comunitario, Centro de Educación Militar (CEMIL), Bogotá.

Correo: mcanesto@gamail.com; Correo: juantellez19811ahotmail.com
} 
installed along the region's oil pipelines, produce environmental damage that adversely affects the economy of the regions and violates the population's fundamental rights. So, it is worth asking: What alternatives would be the most viable to mitigate this situation? The theoretical approach of some sources that have dealt with this problem allowed us to answer the previous question. This comparative descriptive study has an inductive (top-down) approach. Various social, environmental, and safety alternatives were proposed to mitigate the environmental impacts caused by illegal refineries, depending on the conclusion reached.

Keywords: Environmental impact, oil, illegal refinery.

\section{Introducción}

La región del Catatumbo, ubicada al norte del departamento de Norte de Santander, en la frontera con la República Bolivariana de Venezuela, es un remanente de la selva húmeda tropical que abarca también las estribaciones de la cordillera Oriental. Ha sido una región rica en diversidad biológica de la cual se han extraído variedad de recursos, principalmente petróleo, madera y carbón. Habitada ancestralmente por los indígenas Barí, ha sufrido procesos acelerados y desorganizados de colonización, principalmente por la búsqueda de recursos petroleros y hoy por el cultivo de coca (Defensoría del Pueblo, 2016, p. 3).

El problema denunciado por la Defensoría del Pueblo obedece a la proliferación de Grupos Armados Organizados (GAO), el crecimiento exponencial de cultivos ilícitos en el departamento Norte de Santander y la escasez de gasolina en el vecino país de Venezuela, son factores que han incidido en la problemática encontrada sobre la cual no se hallan estudios recientes. Sin embargo y como antecedentes, existen algunos documentos que constituyen una voz de alerta sobre lo que podría ocurrir y que, de hecho, está ocurriendo en esta región del país.

Además, y de acuerdo con la Oficina de las Naciones Unidas contra la Droga y el Delito (UNODC, por sus siglas en inglés), en el informe de monitoreo en el lapso del 2019-2020, publicado en julio del presente año, sobre los cultivos ilícitos en el territorio nacional, menciona que:

Los cultivos de coca en el Catatumbo vienen subiendo continuamente desde el 2010, cuando se reportaron 1.889 ha; en el 2019 se repor$\tan 41.749$ ha. Este aumento hace que, en el 2019, Norte de Santander 
desplace a Nariño como el departamento más afectado por cultivos de coca. Tibú (Norte de Santander) es el municipio con más coca en Colombia (cerca de 20.000 ha), pero en la lista de los diez municipios más afectados por cultivos de coca se encuentran otros tres municipios de este núcleo: Sardinata, El Tarra y Teorama. E1 81\% de la coca en Catatumbo se encuentra en zonas permanentemente afectadas durante los últimos diez años, las cuales ocupan el $46 \%$ del área afectada. Solo el $13 \%$ del territorio alcanzó la categoría de abandono, lo que indica que el fenómeno está fuertemente arraigado en el territorio. (Oficina de las Naciones Unidas contra la Droga y el Delito - UNODC - y Gobierno de Colombia, 2019, p. 81)

Se deduce de este informe de la UNODC, que los diferentes actores ilegales que intervienen en la siembra, cultivo y vigilancia de los cultivos, producen el producto final de su inversión, esto es, el clorhidrato de cocaína, lista para ser enviada a aquellos países con mayor demanda.

Asimismo, y desde la perspectiva de Fayad Sanz (2014):

Los impactos de la explotación petrolera en los conflictos armados se derivan de variables que van desde el orden internacional hasta el ámbito local. En el ámbito internacional, estos impactos son consecuencia de un modo particular de extraer este recurso, un modelo, que obedece a intereses de potencias militares y económicas. En lo local, dicho modelo se expresa de acuerdo con características socioeconómicas, políticas y hasta culturales que terminan por influir en los conflictos armados preexistentes, reconfigurando las fuerzas de los sujetos inmersos en ellos y acentuando las causas estructurales que dieron lugar al nacimiento de ese conflicto. Con frecuencia se habla de que el petróleo es una "maldición", como si detrás de la extracción de este recurso no existieran empresas, gobiernos y ciudadanos que deciden explotar y aprovechar este recurso natural bajo un determinado modelo. (p. 4)

Siguiendo esta línea, Fayad Sanz (2014), citando a Mary Kaldor y Karl Terry, estos argumentan que la actividad petrolera hace más fuertes los conflictos, incluso cuando parece contribuir al fortalecimiento del Estado central. Según los autores, el dominio territorial a través de la presencia de fuerzas armadas, que en las "viejas guerras" eran sinónimo de 
victoria, en las nuevas guerras del petróleo resulta exacerbando el conflicto. Es decir, a mayor fuerza pública alrededor de los enclaves petroleros, mayor inseguridad (Kaldor y Karl, 2007).

Una vez establecido el "enclave petrolero" en países con conflictos latentes, la necesidad de acceder a los recursos lleva a quienes quieran obtener sus beneficios a tomar partido de uno u otro grupo con poder y legitimidad en el territorio (Klare, 2007, p. 26).

Además de lo anterior, en un informe de la Fundación Panamericana para el Desarrollo (Fupad) y la Agencia Nacional de Hidrocarburos (ANH), tratan sobre la alteración del orden público en departamentos priorizados, entre estos Norte de Santander y su región del Catatumbo.

En su trabajo de campo, el equipo consultor y de acuerdo con la metodología propuesta por la $\mathrm{ANH}$, pudieron observar que la mayor complejidad frente a la temática de orden público se encuentra en los departamentos de Santander y Arauca, relacionados con una fuerte oposición social y presión por parte de los grupos armados ilegales (Fundación Panamericana para el Desarrollo - Fupad-y Agencia Nacional de Hidrocarburos -ANH-, 2017, p. 8).

Las citadas entidades afirman textualmente que
Es necesario aclarar que el conflicto en los territorios cambió su naturaleza, pasando de una guerra irregular a un problema de delincuencia dominado principalmente por el cultivo, la transformación y la comercialización de la hoja de coca, especialmente en la zona del Catatumbo y en los departamentos de Putumayo y Caquetá. (Fupad y ANH, 2017, p. 8)

Aclarando que, sin embargo, la presencia de múltiples economías ilegales en las zonas, que van más allá del narcotráfico, como la minería ilegal, el tráfico de gasolina, el contrabando, la trata de personas, la explotación sexual y el tráfico de migrantes, también debe tenerse en cuenta al momento de realizar un análisis de contexto del territorio para la implementación de proyectos y el establecimiento de las necesidades en la zona, pues no solo puede obedecer a requerimientos de las comunidades, sin un contexto establecido o sin que se pueda crear proyectos a largo plazo que sean sostenibles, los cuales llegarían a tener un bajo impacto, como hacer una cancha de futbol si no se cuenta con un programa deportivo y de articulación con las comunidades para el esparcimiento, el deporte y la sana utilización del tiempo libre. 
Más adelante, Fupad y ANH mencionan que

Asimismo, debe observarse en estos territorios el riesgo al que están expuestos los migrantes venezolanos de ser reclutados por los grupos armados al margen de la ley, como también su incorporación a las economías ilegales mediante el suministro de su mano de obra. Para las mujeres venezolanas el riesgo de explotación sexual y de feminicidios es mayor, en el marco de su vinculación al trabajo sexual y a las economías ilegales. (p. 9)

Teniendo en cuenta lo anterior, y asociándolo al contexto, profesores de la Universidad de los Andes, se refieren al conflicto social que genera la explotación de petróleo en Colombia, lo que a modo de ver de estos investigadores, no constituye novedad, puesto que diversos casos en todo el mundo dan cuenta de las tensiones políticas, económicas y sociales asociadas a la explotación y distribución del llamado "oro negro” (Rettberg y Prieto, 2018, p. 135).

Respecto a este párrafo, se argumenta que en todo el mundo no existen los actores armados ilegales que operan en Colombia dedicados al hurto del crudo como insumo en la producción de cocaína.
$\mathrm{Al}$ igual que en otras partes del mundo, el petróleo colombiano ha generado grandes ingresos para el Estado y para actores sociales específicos, pero también ha sido vinculado a tensiones políticas, económicas $\mathrm{y}$ sociales, corrupción y debilidad institucional. El temor al despilfarro de regalías, el peligro de contraer la "enfermedad holandesa" y los múltiples modos de relación entre el petróleo, el conflicto armado y diferentes formas de criminalidad periódicamente dominan los análisis noticiosos de la economía colombiana. (Rettberg y Prieto, 2018, pp. 135-136)

Aclarando que se entiende por enfermedad holandesa,

el proceso mediante el cual las bonanzas externas, como las provenientes del petróleo, llevan a la contracción acelerada de sectores productores de bienes comercializables, como resultado del "crecimiento de la razón de los precios de los bienes no transables y los transables, y de la transferencia de [...] mano de obra y capital desde los últimos hacia los primeros. (Puyana y Thorp, 1998)

Sobre la región del Catatumbo, también la Defensoría del Pueblo en la 
Resolución 46 del 11 de diciembre del 2006, indicaba:

Que la región del Catatumbo es muy rica en diversidad biológica, con abundantes recursos de flora y fauna, principalmente en las zonas donde se conservan la selva andina.

Que la región del Catatumbo es un enclave importante por su localización fronteriza, gran parte de la cual es reserva forestal.

Que la extracción histórica de los recursos naturales no ha traído el bienestar esperado a la población y, por el contrario, ha aumentado la pobreza en la región.

Que los impactos ambientales se evidencian en la pérdida de cerca de 200.000 ha de bosque, el cambio del uso del suelo, los procesos erosivos, la alteración de la regulación hídrica, la pérdida de biodiversidad. (Defensoría del Pueblo, 2006, p. 1)

Por otra parte, el conflicto armado en Colombia implica que Grupos Armados Organizados practiquen actividades ilegales como la minería, refinería de petróleo y elaboración de pasta base de coca para tener un usufructo proveniente de la explotación o hurto de recursos naturales, en territorios de difícil acceso y con poca presencia del Estado, como en Tibú.

Consecuente con lo anterior, la Oficina de las Naciones Unidas contra la Droga y el Delito (UNODC, por sus siglas en inglés), ya citada, afirma según la perspectiva de Suárez, que las dinámicas en la región se han visto potenciadas por diferentes actividades criminales que se congregan y promueven la producción ilícita de drogas, como el hurto de crudo al oleoducto Caño Limón-Coveñas, el cual es usado para la fabricación artesanal de sustancias químicas (hidrocarburos similares a la gasolina). Estas sustancias se trafican en la región y se emplean en el procesamiento de la hoja de coca y en la extracción del alcaloide. Además, como consecuencia de esta práctica, se derivan graves secuelas ambientales por las piscinas empleadas para la destilación artesanal del crudo y los eventuales derrames ocasionados por el hurto (Suárez, 2020).

Toda esta información, es necesario tenerla en cuenta, puesto que "existen varias problemáticas como la generación de residuos aceitosos (borras) en la industria petrolera puede ser considerada como una pérdida del proceso y un mal aprovechamiento de la materia prima empleada" (Torres-Cervera, 2014, p. 2). 
Algunos de los compuestos presentes en las borras son transferidos al aire en forma de compuestos orgánicos volátiles, debido a las condiciones de alta temperatura usualmente existentes en las zonas de extracción. Por otro lado, no existen evidencias de que los microorganismos nativos efectivamente tengan capacidad de degradar los compuestos contaminantes. Posteriormente, aunque así sea, las condiciones de biodegradación reales no son las mismas determinadas en los ensayos de laboratorio, ni permanecen constantes en un mismo sitio, puesto que el mezclado es deficiente, las condiciones de temperatura y climatológicas varían, no se llegan a efectuar pruebas adecuadas para determinar el nivel de biodegradación de los diversos contaminantes (principalmente hidrocarburos), por lo que el tiempo de biodegradación apropiado es incierto (Benítez et al., 2004).

Además, y de acuerdo con lo que menciona Lastra Mier (2015) en su artículo, apoyado en un importante número de fuentes, indica que los documentos expedidos por el Ministerio de Medio Ambiente y el Programa de las Naciones Unidas para el Desarrollo, la degradación del bosque, suelos, agua y biodiversidad en Colombia, tiene muchas causas, entre ellas se destacan las actividades agropecuarias extensivas, la expansión de la minería, los cultivos de uso ilícito y la extracción de maderas tropicales.

De todas las anteriores, la expansión de la ganadería y los monocultivos se considera en el mundo, la segunda causa de pérdida de biodiversidad (luego de las invasiones biológicas) y ha tenido impacto preponderante en la deforestación y degradación de los bosques y del suelo del país. En Colombia, hasta 1990, el bosque cubría el 56,5\% del territorio nacional continental y en el 2010 el 51,4\%, de tal manera que casi el $5 \%$ de los bosques en el país han desaparecido en menos de dos décadas, coincidiendo con su transformación principalmente en praderas para ganadería y áreas agrícolas (Lastra Mier, 2015, p. 64).

A todo lo anterior, se le ha de agregar los deterioros derivados del conflicto armado, tales como los atentados terroristas contra la infraestructura petrolera, la deforestación para cultivos ilícitos, la contaminación por pesticidas para tratar de erradicarlos, la invasión consecuente a su abandono cuando ya no son productivos, entre otros. Atentados terroristas contra la infraestructura petrolera.

A este segmento hay que agregarle los daños causados por los robos que se 
hacen directamente en los oleoductos nacionales por parte de grupos armados. Esta acción se da mediante la instalación de válvulas en los oleoductos del país, generando desde el 2002 a la fecha (2015) la pérdida de 9,3 millones de barriles, de los cuales el $70 \%$, es decir 6,5 millones de barriles, han sido derramados en ecosistemas generalmente frágiles, generando daños ambientales de incalculable valor y que además difícilmente podrán ser reparados.

Expuesto a grandes rasgos el problema y las consecuencias derivadas de este ilícito, el presente documento tiene como objetivo general realizar un estudio comparativo entre la refinería legal e ilegal, con el fin de describir, explicar y proyectar los impactos ambientales causados por la refinería al margen de la ley, en Tibú.

\section{Metodología}

Para llevar a cabo esta investigación, se hizo un estudio descriptivo comparativo dividido en cuatro fases (figura 1): la primera, es la preparación del caso estudio por medio de la búsqueda de antecedentes y soportes bibliográficos mediante la revisión y recopilación de información de fuentes primarias (registros fotográficos de operaciones militares e informa- ción clasificada del Ejército Nacional) y secundarias (bases de datos de universidades y documentos de empresas del sector petrolero). La segunda, es el análisis de la información donde se hace la respectiva selección e interpretación de los documentos para la realización del artículo. La tercera, es la determinación de los impactos ambientales basados en la generación emisiones atmosféricas, vertimientos y residuos de una refinería legal y así, por medio de una evaluación cualitativa de impactos ambientales (matriz de Leopold, modelo simple de dos dimensiones), valorar los impactos (bajo, moderado y alto) y el tipo de afectación positiva, negativa o neutra para el ambiente. Teniendo en cuenta los resultados de la evaluación en la fase cuatro, se plantearon alternativas que contribuyan a la mitigación y de los impactos basadas en información de proyectos petroleros implementados en Colombia.

Como se enunció en el resumen de este documento, se realiza un estudio comparativo entre la refinería ilegal y su abismal diferencia con la refinería legal.

\section{Refinería legal}

Ya en la situación legal, debe tenerse en cuenta la normatividad colombiana 
que regula la explotación de hidrocarburos y sus derivados.

Como lo manifiestan López et al. (2013, p. 18), funcionarios del Banco de la República, quienes manifiestan que luego de la promulgación de la nueva Constitución Política en la que se definieron las funciones de los agentes y del Estado, en 1999 con la expedición del Decreto 2152 (Presidencia de la República, 1992) se modificó la estructura or- ganizativa del sector de minas y energía y se determinó que estaría conformado por el Ministerio de Minas y Energía (MME), unidades administrativas especiales, establecimientos públicos y entidades vinculadas. En el 2003, con la creación de la Agencia Nacional de Hidrocarburos (ANH), se concluyó el diseño actual de la estructura organizativa del sector minero-energético.

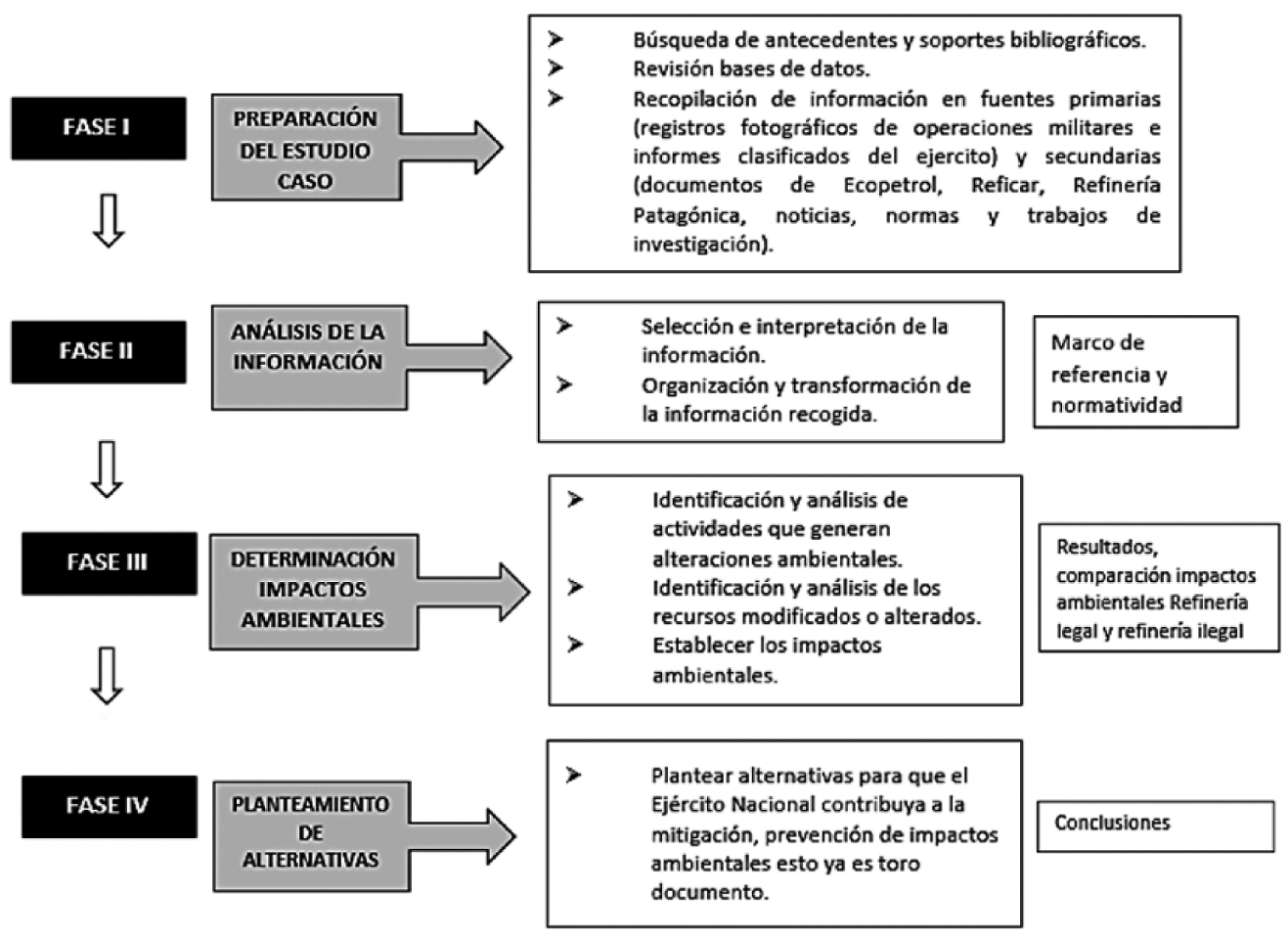

Figura 1. Fases de la metodología descriptiva. Diseño de la investigación

Fuente: elaboración propia. 

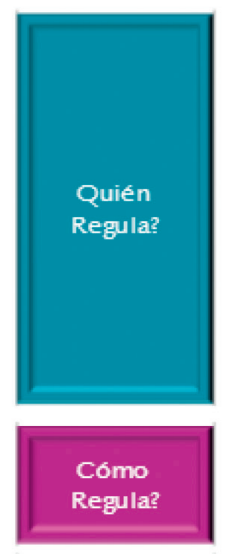

Quiénes participan?

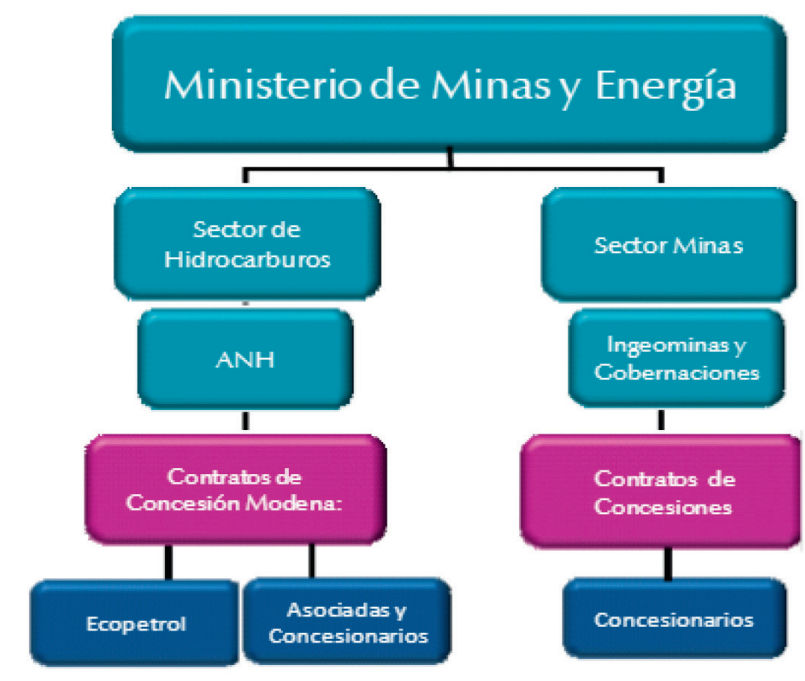

Figura 2. Principales actividades del sector

Fuente: López et al., p. 17.

Como lo demuestra la figura 2, la actual estructura organizativa de las entidades públicas vinculadas al sector petrolero nacional, está inscrita en el esquema funcional definido para el conjunto de la actividad minero-energética del país. Este esquema está establecido conforme a lo estipulado en la Constitución de 1991, según la cual el Estado no interviene de manera directa en la actividad económica, pero sí establece normas y reglas claras de participación a los agentes. En este sentido, por medio de las diferentes instituciones relacionadas con el sector minero, el Estado crea y promueve las condiciones para que los agentes participen en dicha actividad. Por tanto, el Estado desempeña un papel de facilitador, promotor y fiscalizador de la actividad minera (López et. al., 2013, p. 18).

El Decreto 1076 del 2015, define el impacto ambiental así: "Cualquier alteración en el medio ambiental biótico, abiótico y socioeconómico, que sea adverso o beneficioso, total o parcial, que pueda ser atribuido al desarrollo de un proyecto, obra o actividad" (Presidencia de la República, 2015). De acuerdo con esta definición, afirma Vargas (2020, p. 29), "un impacto ambiental es un cambio en cualquiera de los componentes del ambiente producido por una acción o actividad humana con implicaciones ambientales".

Lo anterior significa que cualquier actividad para la explotación de hidro- 
carburos y minería por fuera de la aprobación de estas actividades, está por fuera de lo legal.

En cuanto a las normas ambientales, Colombia posee un sólido y robusto cuerpo normativo, como lo dan a conocer Zaride y Ávila (2014), de la Escuela de Administración de Negocios (EAN).

\section{Refinería ilegal}

Es aquella instalación en la que procesan el crudo de forma artesanal, el cual proviene del hurto en los oleoductos a través de la instalación de válvulas para producir combustible de bajo octanaje, color verdoso y de menor precio llamado "Pata de Grillo", para hacer la respectiva comercialización en laboratorios que producen pasta base de coca. La explotación ilegal se practica en las selvas colombianas como las localizadas en Norte de Santander, Cauca y Nariño, puesto que son lugares con poca presencia del Estado y con condiciones topográficas de difícil acceso.

Por otra parte, debe tenerse en cuenta el significado que tiene la actividad ilegal que expone el proyecto de ley de 2018, "por medio del cual se prohíbe en el territorio nacional la exploración y/o explotación de los Yacimientos No Convencionales (YNC) de hidrocarburos y se dictan otras disposiciones" (Congreso de la República de Colombia, 2018).

Entre las otras disposiciones, señala:

Artículo 1. Prohibición. Prohíbase en el territorio nacional la exploración y explotación de los Yacimientos No Convencionales (YNC) de hidrocarburos como medida de protección del medio ambiente y la salud, y para prevenir conflictos socioambientales asociados a estas actividades.

Artículo 2. Principios. Para los fines de la presente ley deberán aplicarse los principios contenidos en el artículo 1 de la Ley 99 de 1993, el artículo 3 de la Ley 1523 de 2012, la declaración de Río de 1992 y los tratados, convenios y protocolos internacionales sobre medio ambiente y derechos humanos [...].

Téngase en cuenta que este es un proyecto de ley, el cual no se encuentra publicado en el Diario Oficial, debido a múltiples causas, como la controversia que existe entre autorizar el fracking o no autorizarlo, entre otras.

\section{Las prácticas ilegales}

Frédéric Massé y Johanna Camargo, del Observatorio Internacional (DDR Ley de Justicia y Paz - CITpax Colombia), 
son de los pocos autores que se refieren a las prácticas ilegales de la explotación del crudo en Colombia. Según estos autores, los actores armados ilegales han privilegiado su participación como gestores de procesos extractivos de minería en el país, también han tenido cierta participación en el robo de combustibles o como gestores en la cadena de procesamiento de crudo. Estas actividades compensan de cierto modo sus intereses sobre el sector, sin necesidad de disponer del complejo dispositivo de infraestructura para la extracción de crudo. En casos como el contrabando de combustibles, los beneficios económicos suelen ser inmediatos, producto de su gestión directa o del cobro de cuotas a terceros para permitirles desempeñar ese tipo de actividades. En el segundo caso, mediante la refinación de crudo hurtado a las empresas petroleras, los grupos ilegales reducen los costos de adquisición de gasolina, insumo necesario para el procesamiento de estupefacientes (Massé y Camargo, 2012, p. 15).

Estos autores, citando una noticia radial, indican que en el Norte de Santander sucede algo similar. En el sector de Tibú, donde también coinciden cultivos de coca, la Fuerza Pública realiza operativos para desmantelar refinerías clandestinas, donde llega el crudo hurtado por las FARC al Oleoducto Caño Limón-Co- veñas. Allí, el crudo es destilado por la guerrilla, obteniéndose combustible puro para el procesamiento de la hoja de coca producida en el norte y centro del departamento (Caracol Radio, 2012).

Tanto el documento de CITpax como la noticia de Caracol Radio, se publicaron en el 2012, cuando aún no se había firmado el proceso de paz con esta guerrilla; pero ahora operan sus disidencias, ELN y otros grupos armados organizados, en el departamento Norte de Santander.

A continuación, se presenta el procesamiento de una refinería ilegal, de elaboración propia, la cual se basa en el estudio y análisis de fuentes.

La autoría de la figura 3, como la siguiente, se basa en el conocimiento que tiene uno de los autores de la presente investigación quien, por razones de su cargo, pudo comprobar presencialmente, la manera como se hurta el crudo y el proceso que se sigue para su refinamiento, hasta convertirlo en gasolina con destino al procesamiento de la hoja de coca hasta lograr transformarlo en clorhidrato de cocaína y su posterior comercialización en mercados del exterior o bien, la de menos calidad dentro del territorio nacional. Con la salvedad de que esta actividad no generó estudios técnicos sobre el impacto, como lo reafirma más adelante. 


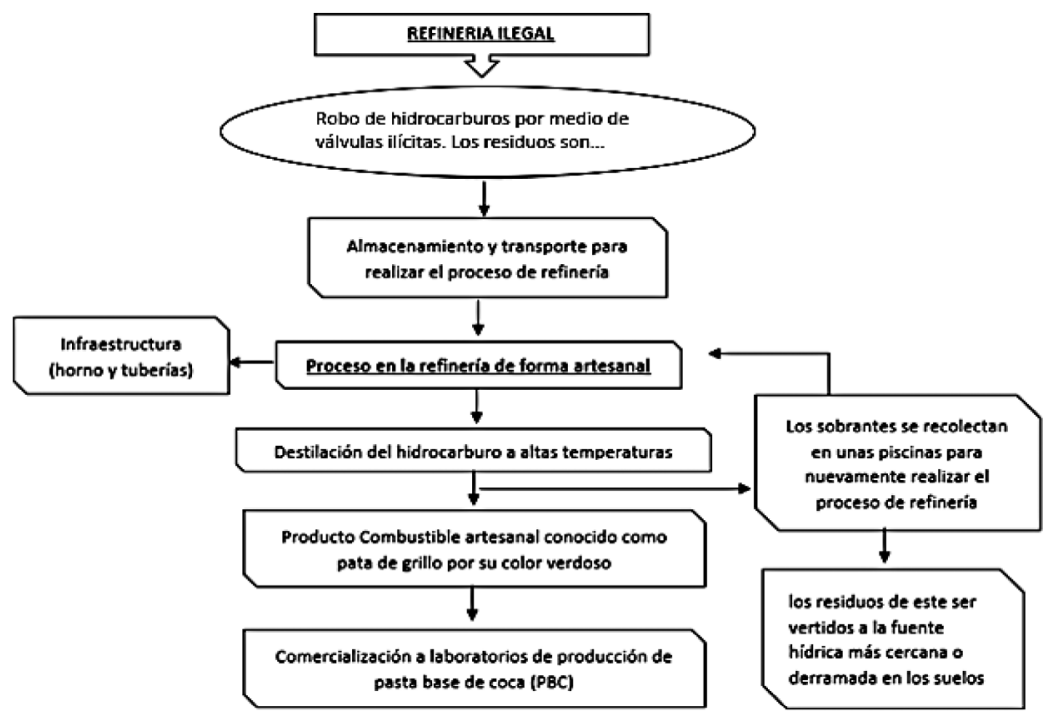

Figura 3. Diagrama de flujo de la operación de una refinería ilegal

Fuente: elaboración propia.

\section{Procesamiento de una refinería ilegal}

Para complementar el diagrama de flujo, se visualiza en la figura 4 el registro fotográfico de una refinería ilegal localizada en Tibú, Norte de Santander, obtenido en operaciones militares.

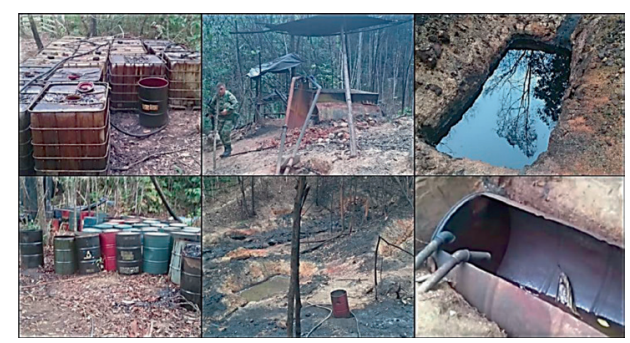

Figura 4. Registro fotográfico de una refinería ilegal localizada en Tibú - Norte de Santander Fuente: elaboración propia.
La metodología empleada y que conduzca a resultados, está basada en la directa observación de una refinería ilegal del municipio estudiado. Igualmente, la recolección y análisis de fuentes permitió conocer las consecuencias que trae para el medio ambiente esta actividad realizada por personas pertenecientes a organizaciones consideradas como Grupos Armados Organizados (GAO), entre estos el ELN, con el propósito de refinar el crudo para el proceso de la coca. Aspecto este sobre el cual vale la pena detenerse brevemente, no sin antes aclarar que el trabajo de campo realizado por el coautor de este documento, se limitó a observar y comprobar, según la figura 4 , y no hacer tipologías o 
indicadores técnicos del sector estudiado; lo cual requiere estudios avanzados sobre el comportamiento que tiene en el medio ambiente (suelos, flora, fauna), las actividades ilegales como lo es el hurto de petróleo a las empresas que obran de acuerdo con la normatividad vigente en Colombia que, además, atienden lo señalado por organismos internacionales sobre el particular. Resumiendo, el presente documento se basa en su totalidad en fuentes bibliográficas obtenidas vía virtual.

A partir de lo anterior y según el trabajo de grado presentado por Eveling Dayana Rodríguez Escalante (2018) ante la Universidad Libre, seccional Cúcuta, este refleja un esmerado estudio técnico y de campo en el que se comprueba lo que el coautor de este documento pudo presenciar.

En efecto, Rodríguez Escalante demuestra gráficamente el grave impacto que producen los derramamientos y hurto de petróleo; además, el respectivo estudio técnico sobre los 18 incidentes estudiados por esta autora, en gran parte sobre el hurto de este hidrocarburo.

Sobre el hurto de petróleo, Rodríguez afirma que durante el 2017 se presentaron nueve incidentes ocasionados por terceros, igualmente que en el 2018, ocasionados por pegas de válvulas y grapas en las líneas de transferencia, perforación en las tuberías, manipulación en los tanques y piscinas de almacenamiento y hurto de las tuberías por donde se traslada el crudo (Rodríguez, 2018, p. 104).

Como se afirmó, esto se debe, básicamente, al empleo del crudo en el procesamiento de la coca, como lo hace saber Ricardo García Rocha quien cita en su texto, a la Oficina de las Naciones Unidas contra la Droga y el Delito (UNODC, por sus siglas en inglés), que en una de sus publicaciones anuales informa sobre el monitoreo que hace sobre los cultivos ilícitos en Colombia, con el aval del Gobierno colombiano, por medio del Sistema Integrado de Monitoreo de Cultivos Ilícitos (SIMCI): la fase agrícola de la coca culmina con el beneficio agroindustrial de la cosecha; es decir, la extracción de base y pasta, la cual corresponde a una tecnología muy sencilla, realizada generalmente en finca por los propios agricultores para facilitar el acopio de la cosecha. Allí se extraen, en promedio, 1,6 kilos de pasta y 1,5 kilos de base por tonelada de hoja de coca, aunque se registran coeficientes superiores a 1,7 kilos en Putumayo, Caquetá y la Orinoquía (Oficina de las Naciones Unidas contra la Droga y el Delito UNODC-, 2006, p. 29). 


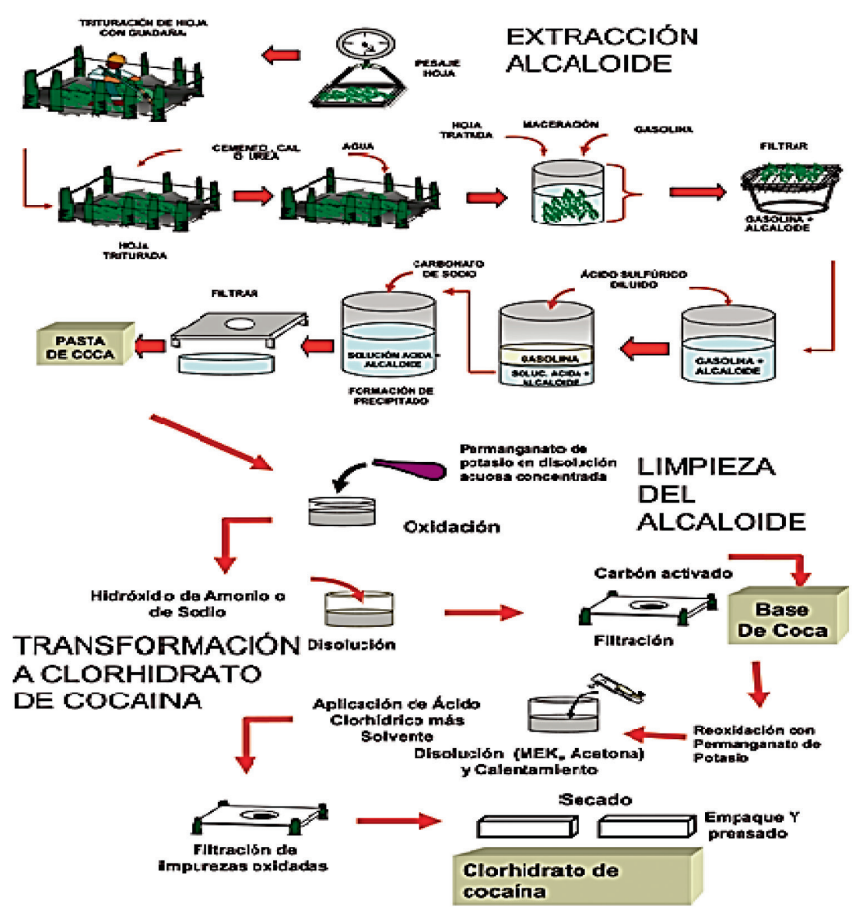

Figura 5. Procesamiento de extracción de pasta, base y cocaína en Colombia Fuente: Dirección de Antinarcóticos (DIRAN) y SIMCI. Adaptación propia de Rocha (2011, p. 59).

Según Rocha (2011): “La pasta es un derivado de la coca necesaria para la obtención de la base, cuyo proceso comprende varias etapas y variantes en el uso de insumos" (pp. 57-58).

A continuación, se describe someramente uno de los procedimientos más comunes, el cual comprende la extracción y limpieza del alcaloide y su transformación a clorhidrato de cocaína.

Como lo menciona Rocha en su texto: "por campesinos en finca", cierto, bajo la estricta vigilancia de los grupos ilegales interesados en este estupefaciente. Así las cosas, unido a los derrames provocados en los oleoductos se suma este rudimentario proceso, donde el empleo de la gasolina es de primera necesidad.

Con base en las anteriores apreciaciones (siembras de coca y proceso para convertir la planta como clorhidrato de cocaína), están los efectos colaterales que provocan en el medio ambiente, tanto los derrames como el refinado de petróleo en los lugares donde tienen sus laboratorios. 
Tabla 1. Indicadores de emisiones atmosféricas, generación de vertimientos y residuos de la actividad de refinación

\begin{tabular}{|c|c|c|c|c|c|}
\hline & Parámetro & Unidad & Valor & 元 & Valor* \\
\hline \multirow{5}{*}{ Emisiones } & NOx & Ton/Barril refinado & 0,000045 & \multirow{9}{*}{ 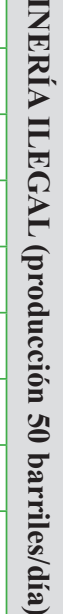 } & 0,00225 \\
\hline & SOX & Ton/Barril refinado & 0,00017 & & 0,0085 \\
\hline & $\mathrm{CO} 2$ & Ton/Barril refinado & 0,042 & & 2,1 \\
\hline & $\mathrm{CH} 4$ & Ton/Barril refinado & 0,000066 & & 0,0033 \\
\hline & Material particulado & Ton/Barril refinado & 0,000023 & & 0,00115 \\
\hline \multirow{2}{*}{ Vertimientos } & Vertimientos industriales & $\mathrm{m} 3 /$ Barril refinado & 0,166 & & 8,3 \\
\hline & Vertimientos domésticos & $\mathrm{m} 3 /$ Barril refinado & 0,0015 & & 0,075 \\
\hline \multirow{2}{*}{ Residuos } & Residuos sólidos no peligrosos & Ton/Barril refinado & 0,00002 & & 0,001 \\
\hline & Residuos sólidos peligrosos & Ton/Barril refinado & 0,000009 & & 0,00045 \\
\hline
\end{tabular}

*Valor: para la producción diaria de 50 barriles. Fuente: Asociación Colombiana del Petróleo (ACP) (2015, p. 20).

Nota: adaptada del Informe de Desempeño Ambiental, de la Asociación Colombiana del Petróleo, diciembre, 2016.

\section{Resultados}

Siguiendo la metodología de tipo descriptivo comparativo, la consulta y el análisis de varias fuentes seleccionadas por su nivel científico, permitieron hacer un estimativo de los indicadores del impacto que causa en el ambiente la refinación del crudo legal e ilegal, basada en la Matriz de Leopold, según se demuestra en la tabla 1.

Dicha matriz es "un procedimiento para la evaluación del impacto ambien- tal y, por tanto, para la evaluación de costos y beneficios" (Ponce, s.f., p. 1).

\section{Paralelo entre una refinería ilegal frente a una legal}

Teniendo en cuenta los impactos ambientales generados por la refinería ilegal, se tomó como referencia los datos teóricos de una refinería en condiciones de legalidad, con el propósito de hacer una aproximación con los datos de campo, sobre los impactos negativos en el medio ambiente, de una refinería ilegal. 


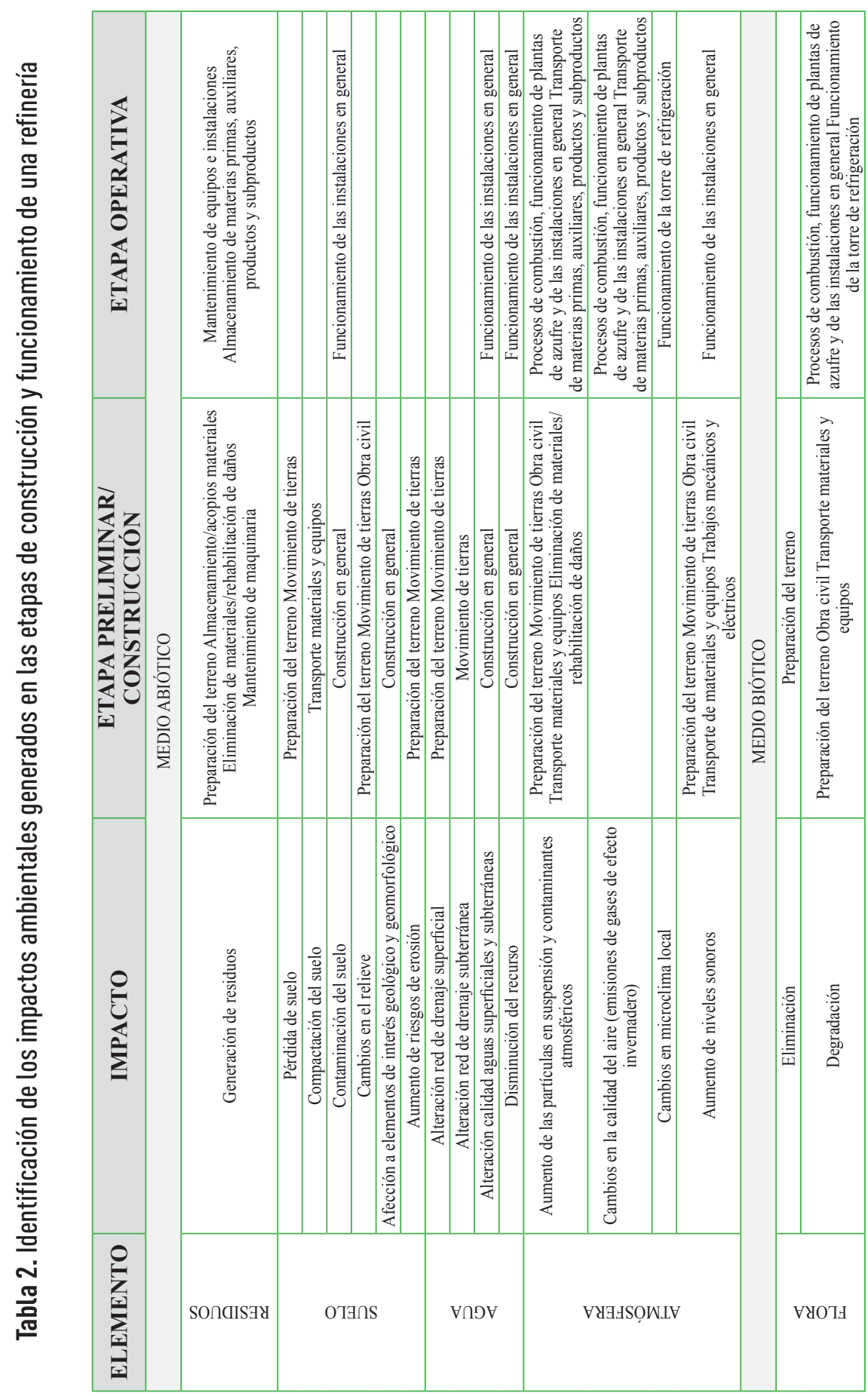




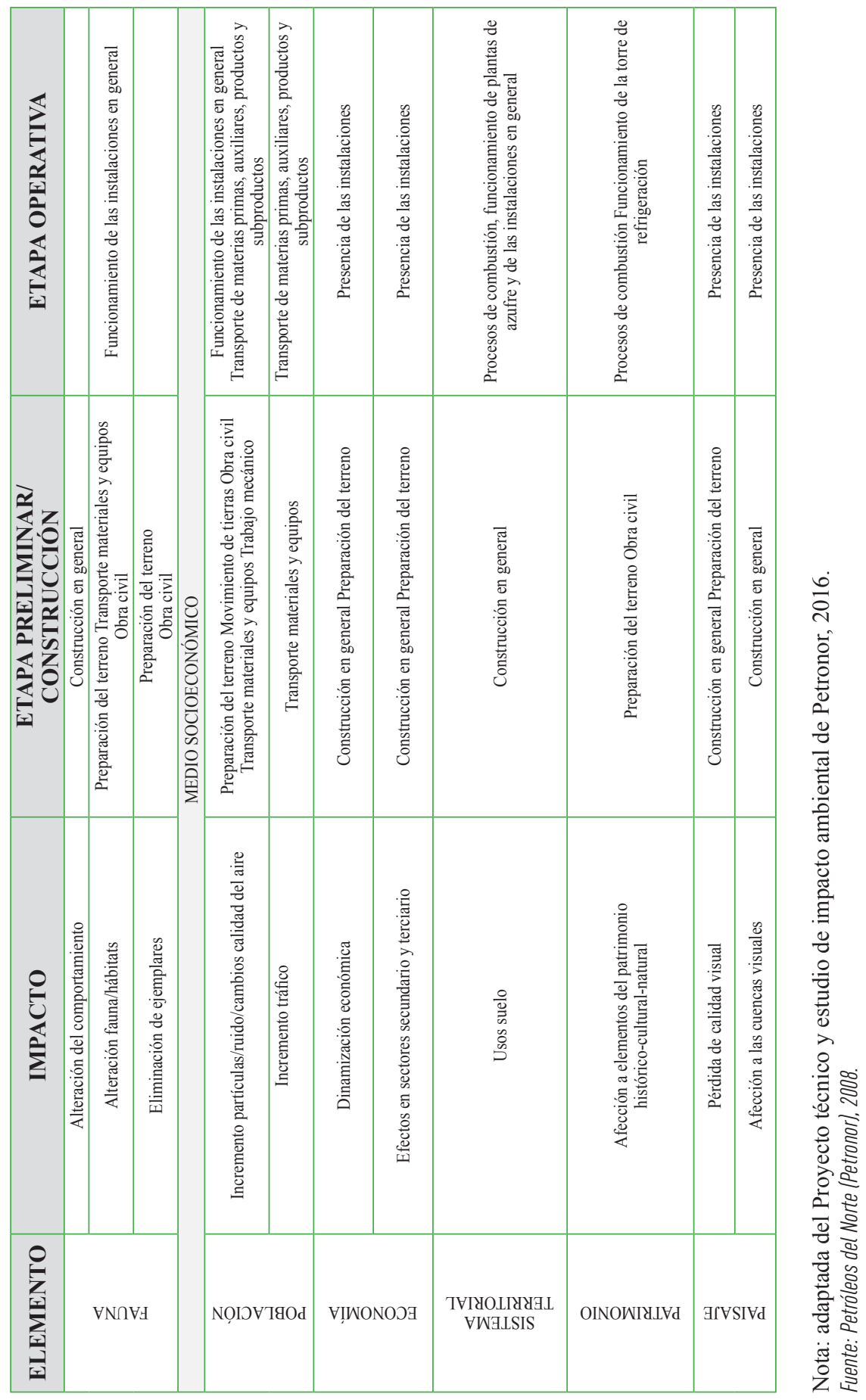


Tomando como referencia un documento de la Asociación Colombiana del Petróleo, se presentan los indicadores de emisiones atmosféricas, vertimientos y residuos generados en la refinación de un barril de petróleo de forma legal. La refinería ilegal genera diariamente 50 veces el valor del indicador. Cabe la pena resaltar que dicho valor depende de la producción diaria de barriles refinados en las instalaciones ilegales.

La identificación de impactos ambientales (tabla 2) se adoptó de las posibles alteraciones que se generan en una refinería legal, teniendo en cuenta las actividades que se ejercen en la fase preliminar, de construcción, operación y desmantelamiento del proyecto y su interacción con el medio abiótico, biótico y socioeconómico.

Posteriormente, se realizó la evaluación cualitativa de impactos ambientales (tabla 3), con base a la información obtenida anteriormente (tabla 2), por medio de la matriz de Leopold (tabla 4), relacionando horizontalmente las actividades del proyecto, construcción, operación y desmantelamiento, y verticalmente los factores ambientales, biótico, abiótico y socioeconómico. Para valorar los impactos se establece una escala que indica si el impacto es bajo, moderado, moderado alto o alto, teniendo en cuenta si este beneficia o perjudica el ambiente.
Tabla 3. Escala de valoración de impactos ambientales

\begin{tabular}{|c|c|c|c|}
\hline \multicolumn{2}{|c|}{ Impacto positivo } & \multicolumn{2}{c|}{ Impacto negativo } \\
\hline Significación & Valoración & Significación & Valoración \\
\hline 1 a 3 & Bajo & -1 a -3 & Bajo \\
\hline 4 a 6 & Moderado & -4 a -6 & Moderado \\
\hline 7 a 9 & Moderado alto & -7 a -9 & Moderado alto \\
\hline 10 a 12 & Alto & -10 a -12 & Alto \\
\hline \multicolumn{4}{|c}{ Impacto Neutro } \\
\hline
\end{tabular}

Nota: adaptada del Estudio de impacto ambiental de la Refinería Patagónica.

Fuente: Refinadora Pavtagónica (s.f.).

En vista de la falta de información técnica acerca de los impactos ambientales causados por las refinerías ilegales, se tomó como referencia los documentos de estudios de impacto ambiental de refinerías que cumplen con la normatividad. Con dicha información se realizó un análisis comparativo para poder estimar y valorar las afectaciones en el medio biótico, abiótico y socioeconómico causado por la construcción, operación y desmantelamiento de una refinería al margen de la ley.

La evaluación de impactos ambientales (tabla 4) es producto del análisis de semejanzas y diferencias entre dos escenarios: el legal y el ilegal, obteniendo una valoración subjetiva de los impactos que generan las refinerías ilegales en Tibú, Norte de Santander. 
Mayra A. Canesto A., Juan G. Téllez C.

\begin{tabular}{|c|c|c|c|c|c|c|c|c|c|c|c|c|c|c|}
\hline \multirow{4}{*}{ 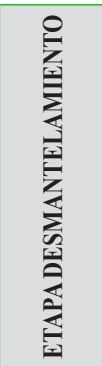 } & 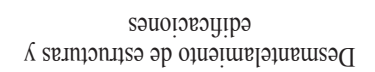 & 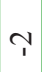 & & in & & & $\nabla$ & $n$ & & & & $a$ & $\infty$ & $\infty$ \\
\hline & 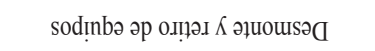 & 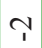 & 7 & 7 & & & & & & & & $a$ & 怾 & \\
\hline & 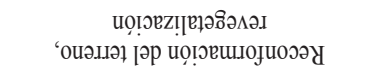 & & $\infty$ & $\infty$ & + & + & 으 & $\stackrel{0}{1}$ & & & 인 & & & \\
\hline & 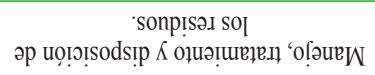 & & & $T^{\prime}$ & $?$ & $?$ & 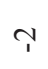 & 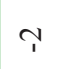 & & & স & & T & \\
\hline \multirow{6}{*}{ 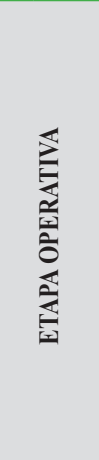 } & 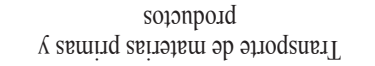 & 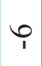 & & & & & & i & & & & & & \\
\hline & 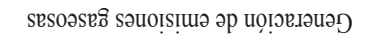 & $\varphi_{1}$ & & & & & & & & & & & & \\
\hline & 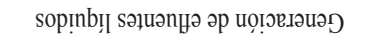 & & & $\hat{i}$ & $\uparrow$ & & & $\varphi_{1}$ & & & & & & \\
\hline & 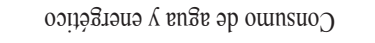 & & & & & 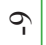 & & & & & & & & \\
\hline & в.qо әр оивш әр врившәб & & & & & & & & & $\infty$ & & & & \\
\hline & 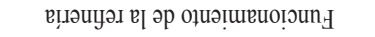 & & & $a$ & & & & $\varphi_{1}$ & & & 9 & & 으 & \\
\hline \multirow{5}{*}{ 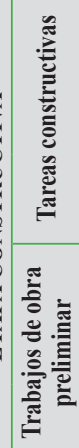 } & в.qо әр оивш әр врившә & & & & & & & & & $\infty$ & & & & \\
\hline & 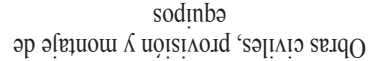 & $?$ & & & & & & P & & & & & & \\
\hline & 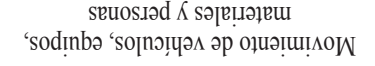 & 广 & & $n$ & & & i & $n$ & & & & & & \\
\hline & so|əns әр оұшә!ш! & † & $T$ & 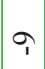 & $T$ & $?$ & & $\varphi_{i}$ & & & T & & & $\infty_{i}$ \\
\hline & 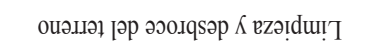 & $n$ & & $a$ & 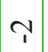 & & 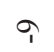 & $\infty$ & & $T$ & i & & & \\
\hline & & 爱 & 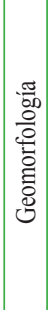 & $\stackrel{\circ}{\stackrel{\Xi}{\Xi}}$ & 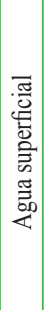 & 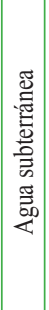 & $\frac{\pi}{\frac{\pi}{I}}$ & 垔 & 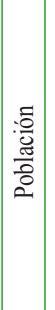 & 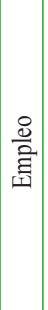 & 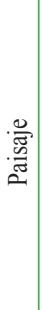 & 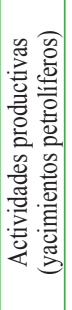 & 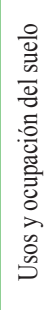 & 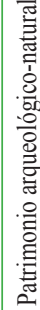 \\
\hline & & & OJI & IL & & & OOI & LỌIg & & DOIV & Ut & ТОЭО & OIJ0 & \\
\hline
\end{tabular}




\section{Análisis de resultados}

La operación, construcción y desmantelamiento de una refinería genera impactos ambientales por inercia; es decir, que cada actividad contribuye a la modificación o alteración en uno de los tres medios: biótico, abiótico o socioeconómico. De acuerdo con la evaluación realizada en la tabla 4 , se concluye que en la etapa de obras preliminares y construcción el impacto es negativo en el medio abiótico, es moderado y moderado alto en el aire; la geomorfología y suelo debido a la generación de material particulado por el alistamiento del terreno y al retiro de la cobertura vegetal del suelo genera erosión, mientras que en el agua el impacto es bajo. En el medio biótico, el impacto es negativo con valoración moderada alta, causado por la deforestación y retiro de la cobertura vegetal, actividad que se hace para adecuar el terreno, repercutiendo en las especies presentes en los ecosistemas, las cuales buscarán refugio en el lugar más cercano. En el sector socioeconómico, la mayor afectación negativa se presenta en el paisaje y patrimonio arqueológico o natural.

En la etapa de operación se evidencia que los valores corresponden a una afectación negativa, moderada, moderada alta y alta en el aire, agua, suelo, flora y paisaje, causadas por la generación de emisiones, residuos, derrame de petróleo y vertimientos; por último, el desmantelamiento y cierre del lugar generan impactos beneficiosos para la industria del petróleo, dado que los Grupos Armados Organizados (GAO) dejan de operar en dicha zona y se disminuyen las pérdidas económicas causadas por el hurto y procesamiento del crudo de forma ilegal; además, debe tenerse en cuenta que esta etapa busca compensar o mitigar los impactos ambientales generados por la construcción y operación de la instalación ilegal de refinerías de hidrocarburos.

\section{Alternativas de mitigación}

Las alternativas establecidas surgen del análisis de la valoración de impactos ambientales valorados en la tabla 4; para su implementación, verificación y control, se deben articular autoridades ambientales, del sector industrial petrolero, la Gobernación de Norte de Santander, el Ejército Nacional y la comunidad, con el propósito de proteger, recuperar y hacer uso sostenible de los recursos naturales de la región.

Las alternativas de mitigación se formulan con el propósito de corregir, recuperar y disminuir los impactos ambientales producidos por la construcción y 
operación de refinerías ilegales en los departamentos de Norte de Santander, Cauca y Nariño; se formulan con base en la evaluación de los impactos ambientales causados en el medio biótico, abiótico y socioeconómico en la etapa de desmantelamiento y cierre de la instalación, puesto que esta actividad la realiza el Ejército colombiano. Las etapas de construcción y operación las ejecutan los Grupos Armados Organizados, a los cuales no les interesa resarcir el daño ambiental causado en dichos lugares, ya que solo realizan esta actividad con el fin de obtener recursos económicos para seguir manteniendo su estructura y elementos.

También, mediante la articulación interinstitucional de los sectores público y privado se involucrará la comunidad en trabajos de sensibilización, dando a conocer las problemáticas ambientales causadas por las actividades ilegales como la refinería que practican los Grupos Armados Organizados en sus territorios; para que se apropien del lugar y participen activamente en procesos de recuperación ambiental. A la vez, se pretende que la población que habita cerca a dichas instalaciones se les brinde información acerca de la riqueza natural que los rodea y la importancia de la conservación y uso racional de los recursos naturales presentes en las selvas colombianas, contribuyendo así al desarrollo sostenible de la región.

La recuperación de los suelos erosionados afectados por el retiro de la cobertura vegetal se lleva a cabo mediante la reforestación, inducción o siembra de especies nativas para Tibú (Norte de Santander) como:

Las Abarco (Cariniana pyriformis), el Cedro (Cedrela odorata), la Ceiba (Ceiba pentandra), el Cañaguate (Tabebuia rosea) y el Pardillo (Cordia Allidora), el Balso (Heliocarpus popayanenses), el Caucho (Castilla elástica), las Jacarandas, los Guamos (Inga sp), el Ninguito (Miconia munutiflora) y los helechos arbóreos: Pochota quinata, Cedrala odorata, Tabebuia rosea, Gmelina arborre. (Camargo, 2017, pp. 40-41)

Para ejecutar esta acción, es necesario establecer el tipo de flora que predomina en el sector y así generar conectividad entre ecosistemas, atrayendo nuevamente algunas especies de fauna que habitaban el lugar, con el propósito de rescatar y recuperar sus principales características.

La reducción de la contaminación del suelo, generada por el derrame de hidrocarburos o sustancias químicas, se efec- 
tuará por medio de tecnologías in situ, puesto que son lugares de difícil acceso. La biorremediación sirve como medida biocorrectiva, la cual consiste en el uso de microorganismos naturales (levaduras, hongos o bacterias) existentes en el medio, que degraden sustancias peligrosas menos tóxicas o inocuas para el medio ambiente, de acuerdo con Alonso (2012). Esta técnica es viable en la zona, pues las condiciones topográficas dificultan el acceso y no tienen vías de acceso vehicular; además, para implementar dicha técnica, debe efectuarse el análisis de contaminantes, la concentración de oxígeno, nivel de nutrientes, $\mathrm{pH}$, temperatura y solubilidad de los contaminantes para determinar si se debe implementar una técnica aerobia o anaerobia para la biorrecuperación del suelo (Alonso, 2012).

Igualmente, otros investigadores se refieren al daño para el medio ambiente por efecto de los suelos contaminados; pues en donde existan fuentes hídricas subterráneas contaminadas por hidrocarburos, se puede ejecutar un proceso de biotransformación natural que reduzca la concentración de contaminantes por medio de la dilución, dispersión, volatilización, adsorción, biodegradación y reacciones químicas que produce el suelo o el agua con la técnica de remediación in situ, de bajo costo, conocida como atenuación natural, que consiste en la utilización de procesos fisicoquímicos de interacción contaminante-suelo y procesos de biodegradación de forma natural (Maroto y Rogel, 2006).

Los cuerpos de agua contaminados por el vertimiento de residuos peligrosos, sustancias tóxicas e hidrocarburos que genera la operación de la refinería, se deben recuperar con la remedición, según Alonso (2012). Se realizaría por medio de la fitorremediación, usando plantas y árboles para depurar aguas y suelos contaminados, donde las plantas cumplen la función de filtrar, descomponer o degradar componentes orgánicos. También, la inyección de aire a cuerpos hídricos contaminados produce burbujas, en las cuales se captan los contaminantes y permite reducir la concentración de contaminantes volátiles. Esta técnica es utilizada para la remediación de aguas subterráneas. Las fuentes hídricas cercanas a la refinería localizada en Tibú, Norte de Santander, son el río Sardinata, río Zulia, quebradas La Cuchara, La Cristalina y Las Indias.

Los impactos atmosféricos como ruido, material particulado y la emisión de gases de efecto invernadero (GEI) disminuyen o se anulan al momento de la desactivación de las estructuras de refinería y está a cargo del Ejército Nacional colombiano. Además, se pueden 
plantear técnicas para la absorción de GEI por medio de plantas y microorganismos.

Las alternativas planteadas anteriormente sirven para recuperar la flora y fauna, especialmente la siembra de especies de vegetación del área, atrayendo la fauna silvestre y generando conectividad entre el sistema afectado con el natural, para reconstituir las características endémicas del área afectada por la construcción y operación de las refinerías ilegales. Dado que son lugares con características de selva y tienen presencia de diversidad ecológica, es más fácil enmendar y recuperar el terreno.

Además, debe contemplarse el manejo adecuado de los residuos peligrosos, como hidrocarburos y sustancias tóxicas, hallados en la operación de desmantelamiento y cierre de la refinería a cargo del Ejército. El cual debe entregar los residuos a gestores autorizados por las autoridades ambientales para el manejo y disposición final de los mismos.

Finalmente, y no menos importante, es el tutorial que presenta The International Council on Clean Transportation (2011), que en la introducción de este documento indica textualmente lo siguiente, que se considera importante para tener en cuenta, ya que aborda:
[...] los principios básicos del refino de petróleo, y que se relacionan con la producción de combustibles ultra bajos en azufre (ULSF), en particular gasolina (ULSG) y combustible diésel (ULSD). Este es el primer producto de trabajo de un completo análisis de la economía de la producción y el suministro de ULSG y ULSD en Brasil, China, India y México, realizado por HART Energy y MathPro Inc. para el Consejo Internacional de Transporte Limpio (ICCT). El propósito del tutorial es (1) proporcionar contexto y un marco organizativo para el análisis general, (2) identificar los factores técnicos que determinan el costo de refinación de la producción de ULSG y ULSD, y (3) facilitar la interpretación de los resultados del análisis. El tutorial aborda:

Fundamentos de la industria del refino de petróleo

- Petróleo crudo y sus propiedades

- Clases de procesos de refinería y configuraciones de refinería

- Propiedades de las corrientes producidas por la refinería ("mezclas de materiales") que componen la gasolina y el combustible diésel

- Opciones de procesamiento de refinería para producir ULSG y ULSD 
Esta guía está dirigida a lectores interesados en la producción de ULSG y ULSD, pero que desconocen las operaciones de refinación en general y el control de azufre en particular.

La refinación de petróleo es un eslabón único y crítico en la cadena de suministro de petróleo, desde la boca del pozo hasta la bomba. Los otros enlaces agregan valor al petróleo principalmente moviéndolo y almacenándolo (por ejemplo, levantando el petróleo crudo a la superficie; moviendo el petróleo crudo de los campos petroleros a las instalaciones de almacenamiento y luego a las refinerías; moviendo los productos refinados de la refinería a las terminales y ubicaciones de uso final, etc.). La refinación agrega valor al convertir el petróleo crudo (que en sí mismo tiene poco valor de uso final) en una variedad de productos refinados, incluidos los combustibles para el transporte. El principal objetivo económico de la refinación es maximizar el valor agregado al convertir el petróleo crudo en productos terminados (The International Council on Clean Transportation, 2011, pp. 1-2)

En pocas palabras, esta entidad brinda información sobre cómo se procesa legalmente el petróleo crudo.
Por otro lado, un grupo de autores ha publicado un trabajo interesante y realiza un estudio comparativo. En el resumen del artículo hacen una comparación del consumo de energía, las emisiones de $\mathrm{CO}_{2}$ y las políticas públicas de dos megaciudades, São Paulo y Shanghái, con el fin de identificar sus políticas de mitigación de emisiones de GEI. Ambas ciudades han experimentado un rápido crecimiento de los sectores de la automoción, lo que ha provocado importantes desafíos de contaminación y emisiones de $\mathrm{CO}_{2}$. São Paulo ha implementado con éxito el etanol y ha fomentado el crecimiento de la flota de vehículos ligeros. Shanghái tiene generación de energía a base de carbón y restringió la propiedad de los vehículos en un intento por reducir las emisiones de GEI, invertidos en transporte público y movilidad eléctrica. En este estudio se adoptó un análisis tabular de datos secundarios, que revela también que São Paulo ha ampliado considerablemente el transporte individual. A pesar de las inversiones en etanol, la ciudad no pudo contener el aumento de las emisiones de $\mathrm{CO}_{2}$ del transporte por carretera. Shanghái invirtió en transporte público e inhibió el transporte individual, pero tampoco pudo contener las emisiones de $\mathrm{CO}_{2}$. Las políticas de 
mitigación y las medidas adoptadas no fueron suficientes para evitar el aumento de las emisiones de $\mathrm{CO}_{2}$ en ambas ciudades. Para reducir las emisiones de $\mathrm{CO}_{2}$ en el transporte, São Paulo y Shanghái deben centrarse en políticas públicas para fomentar el transporte público y limpio y limitar la quema de combustibles fósiles (Costa et. al., 2018).

\section{Conclusiones}

De acuerdo con el título del presente artículo, este tiene como objetivo general hacer un estudio comparativo entre la refinería legal e ilegal con el propósito de describir, explicar y proyectar los impactos ambientales causados por la refinería al margen de la ley, para lo cual siguió una metodología de tipo descriptivo comparativo dividido en cuatro fases (figura 1), para señalar las diferencias entre una actividad ilegal y legal. El documento se orientó a tratar el problema generado por el hurto de petróleo en la región de Tibú, Norte de Santander, por parte de grupos al margen de la ley con el propósito de producir clorhidrato de cocaína con destino al mercado mundial y de consumo interno, actividad que produce un alto impacto ambiental y social, a la vez que genera cuantiosas ganancias para estos grupos, clasificados en la escala del crimen como GAO (Gru- pos Armados Organizados). Asociado a los derrames del mencionado hidrocarburo, están de por medio los derrames provocados por estos mismos grupos a los oleoductos del país, cuyas empresas están constituidas legalmente, siguiendo la normatividad vigente en el país.

La identificación de los impactos ambientales se realizó por medio de la matriz de Leopold, obteniendo como resultado que para la etapa preliminar y la construcción los impactos son negativos tanto para el medio abiótico como biótico. Ante este problema, las alternativas establecidas surgen del análisis de la valoración de impactos ambientales como se demuestra en la tabla 4, considerando que para su implementación, verificación y control, se deben articular autoridades ambientales, del sector industrial petrolero, la Gobernación de Norte de Santander, el Ejército Nacional y la comunidad, con el propósito de proteger, recuperar y hacer uso sostenible de los recursos naturales de la región.

\section{Referencias}

Alonso Riesco, R. (2012). Proyecto de recuperación de suelos contaminados por hidrocarburos. Universidad Autónoma de Barcelona.

Asociación Colombiana del Petróleo. (ACP). (2015). Informe de desempeño ambiental. 
https://acp.com.co/web2017/images/pdf/ publicaciones_e_informes/informe_ambiental/IGA\%202016_WEB.pdf

Benítez, I., Álvarez, R., Escudero, I., Reyes, F. y Rodríguez, A. (2004). Efecto de aditivos nacionales en las propiedades físicas del petróleo crudo cubano. Tecnología Química, 24(1) 43-61.

Camargo Vargas, L. C. (2017). Lineamientos para la gestión de la deforestación generada por los cultivos ilícitos asociados al conflicto armado, en el municipio de Tibú, en el contexto del posconflicto. https://repository.javeriana.edu.co/handle/10554/38071

Caracol Radio. (23 de julio de 2012). Desmantelan en Norte de Santander una refinería clandestina de las FARC. En: Massé y Camargo, Actores armados ilegales y sector extractivo en Colombia. V Informe (2012).

Congreso de la República de Colombia. (2018). Proyecto de ley, "por medio del cual se prohíbe en el territorio nacional la exploración y/o explotación de los Yacimientos No Convencionales (YNC) de hidrocarburos y se dictan otras disposiciones".

Costa, E. et. al. (2018). CO2 emissions and mitigation policies for urban road transportation: São Paulo versus Shanghai. Urbe. Revista Brasileira de Gestão Urbana. On-line version ISSN 2175-3369, 10(supl. 1).

Defensoría del Pueblo. (11 de diciembre del 2016). Situación social y ambiental de la región del Catatumbo - Norte de Santander. Resolución $N^{\circ} 46$.

Fayad Sanz, D. (2014). Petróleo y conflicto armado en Colombia. El caso de Arauca entre 1982 y 1992 (trabajo de grado). Pon- tificia Universidad Javeriana.

Fundación Panamericana para el Desarrollo (Fupad) y Agencia Nacional de Hidrocarburos (ANH). (2017). Análisis de alteración a la seguridad pública y la conflictividad social en el sector de hidrocarburos en Colombia. https://www.anh.gov. co/Seguridad-comunidades-y-medio-ambiente/Estrategia-social/Documents/ Documento\%20ejecutivo\%20Alteraci\%C3\%B3n\%20Orden\%20Publico.pdf

Kaldor, M., \& Karl, T. (2007). Oil Wars. http://economic.free.fr/crisis/ $\% \mathrm{CA} \% \mathrm{~A}$ F\%D3\%CD\%D5\%BD\%D5\%F9\%20 $\%$ D3\%A2\%CE\%C4\%B0\%E6.pdf

Klare, M. (2007). Sangre y petróleo: peligros y consecuencias de la dependencia del crudo (citado por Fayad Sanz).

Lastra Mier, R. (2015). Degradación medioambiental como consecuencia del conflicto armado en Colombia. Universidad del Atlántico. Legem. ISNN: 2346-278, 3(1).

López, E., Montes, E., Garavito, A. y Collazos, M. (2013). La economía petrolera en Colombia (Parte I). Cuadernos de Economía, $\mathrm{N}^{\circ} 692$.

Massé, F. y Camargo, J. (2012). Actores armados ilegales y sector extractivo en Colombia. V Informe (CITpax Colombia y Observatorio Internacional DDR - Ley de Justicia y Paz).

Oficina de las Naciones Unidas contra la Droga y el Delito (UNODC) y Gobierno de Colombia. (2019). Colombia. Monitoreo de territorios afectados por cultivos ilícitos. ISBN digital: 978-958-5554-14-6. 
Oficina de las Naciones Unidas contra la Droga y el Delito (UNODC). (2006). Colombia. Monitoreo de cultivos de coca. En: Rocha García, R., Las nuevas dimensiones del narcotráfico en Colombia (2011) (pp. 57-58).

Petróleos del Norte (Petronor). (2008). Proyecto técnico y estudio de impacto ambiental. https://www.euskadi.eus/contenidos/ documentacion/esia_urf_petronor/es_doc/ adjuntos/esia_urf.pdf

Ponce, V. (s.f.). La matriz de Leopold para la evaluación del impacto ambiental. http:// ponce.sdsu.edu/la_matriz_de_leopold.html

Presidencia de la República. (1992). Decreto 2152.

Presidencia de la República. (2015). Decreto 1076.

Puyana, A. y Thorp, R. (1998). Colombia. Economía política de las expectativas petroleras. Globalización, políticas sectoriales y empleo. En: A. Retteberg y J. Prieto, Conflicto crudo: petróleo, conflicto armado y criminalidad en Colombia. doi:10.30778/2018.09.

Refinadora Patagónica. (s.f.). Estudio de impacto ambiental de la Refinería Patagónica Comodoro Rivadiavia, Provincia del Chabut, Argentina.

Rettberg, A. y Prieto, J. (2018). Conflicto crudo: petróleo, conflicto armado y criminalidad en Colombia. doi:10.30778/2018.09.

Rocha García, R. (2011). Las nuevas dimensiones del narcotráfico en Colombia. ISBN: 978-958-99962-5-6.
Rodríguez Escalante, E. D. (2018). Monitoreo de las afectaciones ambientales originadas por la extracción ilícita del crudo de la infraestructura petrolera en el campo de explotación de hidrocarburos Tibú (tesis de grado). Universidad Libre, seccional Cúcuta.

Suárez, N. (2020). "Pategrillo”, una ventaja inusitada para los laboratorios de cocaina en Colombia. En: UNODC, Gobierno de Colombia.

The International Council on Clean Transportation. (2011). An introduction to petroleum refining and the production of ultra low sulfur gasoline and diesel fuel. https:// theicct.org/publications/introduction-petroleum-refining-and-production-ultra-low-sulfur-gasoline-and-diesel

Torres-Cervera, K. P. (2014). Análisis de los impactos ambientales generados por el tratamiento y disposición final de los residuos aceitosos (borras) generados en los distritos de producción de hidrocarburos (trabajo de grado). Universidad Nacional de Colombia.

Vargas Guarín, L. (2020). Impactos ambientales de la producción petrolera en Colombia y su relación con la innovación tecnológica en los últimos quince años (trabajo de grado). Universidad de América.

Zaride, N. y Ávila, A. (2014). Principales normas ambientales colombianas. ISBN: 78958-756-279-8. 